Cahiers
de a Recherche
sur les Droits Fondamentaux
Cahiers de la recherche sur les droits fondamentaux

17 | 2019

La motivation des actes administratifs. Le droit français à la lumière du droit administratif comparé

\title{
La consécration en clair-obscur de l'obligation de motivation des actes administratifs au Chili
}

\section{Carolina Cerda-Guzman}

\section{(2) OpenEdition}

\section{Journals}

Édition électronique

URL : https://journals.openedition.org/crdf/3820

DOI : $10.4000 /$ crdf. 3820

ISSN : 2264-1246

Éditeur

Presses universitaires de Caen

Édition imprimée

Date de publication : 31 décembre 2019

Pagination : 161-170

ISBN : 978-2-84133-960-0

ISSN : 1634-8842

Référence électronique

Carolina Cerda-Guzman, « La consécration en clair-obscur de l'obligation de motivation des actes

administratifs au Chili », Cahiers de la recherche sur les droits fondamentaux [En ligne], 17 | 2019, mis en ligne le 06 février 2021, consulté le 14 novembre 2022. URL : http://journals.openedition.org/crdf/3820 ; DOI : https://doi.org/10.4000/crdf.3820 


\title{
La consécration en clair-obscur de l'obligation de motivation des actes administratifs au Chili
}

\author{
Carolina CERDA-GUZMAN \\ Maîtresse de conférences en droit public à l'université Paul-Valéry - Montpellier 3 \\ Centre d'études et de recherches comparatives sur les constitutions, les libertés et l'État (CERCCLE, EA 7436)
}

I. Des prémices aux prémisses de l'obligation de motivation des actes administratifs au Chili

A. Les origines fragmentaires de l'obligation de motivation

B. Les fondements constitutionnels et légaux de l'obligation de motivation

II. Des contours incertains de l'obligation de motivation des actes administratifs

A. Un champ d'application aux délimitations encore imprécises

B. Des incidences de l'obligation de motivation encore flottantes

La motivation des actes administratifs est une obligation relativement nouvelle en droit administratif chilien, puisque ce n'est que par une loi de 2003 que cette obligation a enfin été formulée de manière générale ${ }^{1}$. Ce retard peut être attribué à divers facteurs, mais les deux principaux mis en avant sont la forte tradition du secret qui a longtemps marqué le fonctionnement des administrations hispano-américaines et le développement, au Chili, d'une puissante et forte bureaucratie qui faisait peu de cas des doléances des administrés ${ }^{2}$.

La tardiveté de la consécration devrait a priori faciliter sa présentation aux lecteurs non spécialistes du système juridique chilien. Pour autant, il n'en est rien dès lors que l'on traite d'une notion de droit administratif dans le cadre de ce système juridique. Toute analyse d'une notion, concept ou règle de droit administratif au Chili nécessite qu'au préalable on s'attarde quelque peu sur des éléments caractéristiques de cette branche du droit. Certaines de ces caractéristiques sont communes aux droits des pays hispanophones, d'autres, en revanche, sont propres au Chili. Deux d'entre elles sont particulièrement importantes et expliquent en partie pourquoi il peut apparaître comme particulièrement audacieux d'entreprendre une étude de la motivation des actes administratifs au Chili.

La doctrine juridique dans ce pays, comme dans nombre d'autres pays hispano-américains, accorde une place importante, voire démesurée, à l'analyse du droit et de la doctrine étrangère. Cela est notamment le cas en droit administratif. La grande majorité des auteurs renvoie soit à des auteurs étrangers, soit à des normes juridiques étrangères au moment d'étudier une institution ou une

1. Loi no 19.880 du 29 mai 2003 établissant les bases des procédures administratives qui régissent les actes des organes de l'administration de l'État.

2. E. Rocha Fajardo, Estudio sobre la motivación del acto administrativo, mémoire en sciences juridiques et sociales, université du Chili, 2016, 308 p., p. 195-196. 
notion du droit. Le plus courant est le renvoi au droit ou à la doctrine espagnols ${ }^{3}$, latino-américains (qu'il s'agisse d'auteurs argentins ou uruguayens ${ }^{4}$ ), mais aussi à la doctrine et au droit allemands ${ }^{5}$, français ${ }^{6}$ et même parfois britanniques $^{7}$ ou états-uniens ${ }^{8}$. Ce recours important au droit étranger est parfois ouvertement assumé par la doctrine, qui considère que le droit étranger sert de modèle au Chili $^{9}$; pour d'autres auteurs, certains concepts étant par nature universels, il serait dès lors justifié d'avoir recours à des auteurs étrangers pour l'analyse ${ }^{10}$. Vue de France, et notamment si l'on est comparatiste, cette pratique peut apparaître comme très positive. Cependant, le caractère systématique et l'absence de contextualisation de la doctrine et du droit étrangers présentent des risques. Tel que le signale Eduardo Soto Kloss, les auteurs chiliens oublient parfois d'adapter ces données au cadre juridique national, c'est-à-dire aux sources propres chiliennes (notamment les sources indigènes) ${ }^{11}$. Pour illustrer cette «fuite vers le droit étranger ${ }^{12}$, l'auteur prend l'exemple de la définition de l'acte administratif au Chili. Afin de correspondre aux standards étrangers, l'acte administratif a longtemps été défini de manière strictement positiviste par la doctrine chilienne, comme une décision unilatérale d'un organe étatique dans l'exercice des fonctions administratives. Or, cette définition ne correspondait pas pleinement à l'état du droit chilien. En effet, le droit constitutionnel chilien permettait de donner corps à une définition plus substantielle de l'acte administratif, prenant en compte son contenu et surtout sa finalité ${ }^{13}$. Ces désaccords sur la notion d'acte administratif impliquent nécessairement des divergences dans l'analyse des vices de légalité de l'acte et, donc, à terme, sur la nature formelle ou substantielle du vice de motivation, comme il sera vu ultérieurement.

Cette importance du droit étranger au sein de la doctrine administrativiste chilienne pourrait constituer un point de détail si elle n'avait pas eu également des incidences sur la structure même de son contentieux. Influencé par le modèle juridictionnel français, mais également soucieux de préserver les pouvoirs des institutions juridictionnelles déjà en place, le Chili a longtemps hésité à mettre en place une juridiction administrative spécifique pour traiter du contentieux administratif ${ }^{14}$. Aujourd'hui, le contentieux administratif chilien est particulièrement complexe, rendant ainsi difficile la présentation simplifiée du contrôle juridictionnel de la motivation des actes administratifs. Les deux principaux organes actuellement compétents pour contrôler la légalité des actes administratifs sont la Contraloría General de la República (CGR) et les juridictions judiciaires (avec à leur tête la Cour suprême) ${ }^{15}$. La CGR est une instance administrative, consacrée par l'article 98 de la Constitution chilienne ${ }^{16}$. Elle est chargée de contrôler la légalité et la constitutionnalité des actes produits par certains organes de l'administration, notamment les décrets suprêmes du président de la République et les décisions des chefs de service de l'administration de l'État ${ }^{17}$. Ce contrôle étant opéré ex ante, avant l'entrée en vigueur des textes, la CGR n'est pas considérée comme une juridiction administrative à proprement parler. Cependant, elle a acquis un rôle central dans le contrôle de l'administration chilienne et

3. Pour quelques exemples dans les articles portant sur la motivation, voir I. Aróstica Maldonado, «La motivación de los actos administrativos en el derecho chileno ", Revista de derecho de la Pontifica Universidad Católica de Valparaiso, n 10, 1986, p. 499, 503, 505; A. Fermandois Vöhringer, J. Baraona González, «La inexistencia de los hechos que fundamentan un acto administrativo, ¿ constituye un vicio de nulidad?», Revista de derecho de la Universidad Finis Terrae, année VII, nº 7, 2003, p. 85, 86, 92 et 93; A. Vergara Blanco, "La motivación de los actos administrativos", in La Contraloría General de la República y el Estado de Derecho. Conmemoración por su $75^{\circ}$ Aniversario de vida institucional, Santiago du Chili, Contraloría General de la República, 2002, p. 344-349; J. Bermúdez Soto, «Estado actual del control de legalidad de los actos administrativos. ¿Qué queda de la nulidad de derecho público?», Revista de derecho, vol. 23, nº 1, juillet 2010, p. 106-108.

4. I. Aróstica Maldonado, «La motivación...», p. 501; A. Fermandois Vöhringer, J. Baraona González, «La inexistencia de los hechos...», p. 83, 85,89 et 90 .

5. A. Fermandois Vöhringer, J. Baraona González, «La inexistencia de los hechos...», p. 86 et 93; J. Bermúdez Soto, «Estado actual...», p. 104, 106,107 et 119

6. Y sont cités M. Waline, M. Hauriou et même Merlin. Voir I. Aróstica Maldonado, «La motivación... », p. 504; A. Fermandois Vöhringer, J. Baraona González, «La inexistencia de los hechos... », p. 81 et 85; A. Vergara Blanco, «La motivación... », p. 344.

7. I. Aróstica Maldonado, «La motivación...», p. 506 et 508; T. Carrera Briceño, «El deber de motivación de los actos administrativos. El caso de los actos del proceso calificatorio", Revista de derecho administrativo económico, nº 24, janvier-juin 2017, p. 143.

8. A. Fermandois Vöhringer, J. Baraona González, «La inexistencia de los hechos...», p. 80.

9. J. Bermúdez Soto, «Estado actual...», p. 104.

10. A. Fermandois Vöhringer, J. Baraona González, «La inexistencia de los hechos... », p. 91.

11. E. Soto Kloss, «La noción de acto administrativo en el derecho chileno (una perspectiva sustancial)», Revista de derecho público, n 6o, 1996, p. 85-86.

12. Ibid., p. 85. Toutes les traductions de l'espagnol au français ont été faites par l'auteure de cet article.

13. Ibid., p. 86

14. Jusqu'en 1989, la Constitution prévoyait que le contentieux administratif serait confié à une juridiction particulière. Cependant, la mise en place de tribunaux administratifs n'est pas parvenue jusqu'à son terme. Face à cet échec, le choix a été fait de supprimer l'expression "contentieux administratif» de la Constitution, permettant ainsi de lever la barrière constitutionnelle au contrôle de légalité des actes administratifs par le juge judiciaire. Voir C. Cerda-Guzman, "Importation d'objets juridiques et cohérence de l'ordre juridique administratif chilien", Revue internationale de droit comparé, vol. 59, n² 2, 2007, p. 307-332; J. Bermúdez Soto, «Estado actual... », p. 104.

15. Il serait également possible d'évoquer le Tribunal constitutionnel, qui peut contrôler la constitutionnalité des décrets suprêmes du président de la République (articles 93, $9^{\circ}$ et $16^{\circ}$ de la Constitution du Chili), mais son rôle dans le développement du droit administratif est moins direct que celui de la CGR et des juridictions judiciaires.

16. Sur l'histoire et les origines anciennes de cette institution, voir A. Vergara Blanco, «El rol de la Contraloría General de la República: desde el control de legalidad a los nuevos estándares de buena administración », in La Contraloría General de la República. Conmemoración por su $85^{\circ}$ Aniversario de vida institucional (1927-2012), Santiago du Chili, Contraloría General de la República, 2012, p. $103-110$.

17. Loi n 10.336 du 29 mai 1952 portant sur l'organisation et les attributions de la Controlaría General de la República. 
ses avis s'imposent à tous les organes de l'administration de l'État soumis à son contrôle.

Suite au renoncement à créer une juridiction administrative, le contrôle juridictionnel ex post des actes administratifs a été confié aux juridictions de droit commun. Dans la mesure où cette solution avait été vue initialement comme temporaire, le contentieux administratif ne s'est pas construit de manière uniforme mais par des retouches ou ajouts successifs. Actuellement, ce contentieux est structuré autour de différents recours, très nombreux, qui se distinguent selon l'objet de la demande, les violations alléguées, le document contesté et les demandes de réparation. Les trois principaux recours sont le recours en nullité de droit public, le recours de protection et le recours contre les actes des administrations municipales. Chacun de ces recours est entouré de règles procédurales distinctes et produit des effets différents. Dès lors, au moment d'examiner la manière dans la motivation des actes administratifs est contrôlée par les juges au Chili, cette complexité rendra plus ardue toute tentative de généralisation.

Ainsi, bien que l'étude de la motivation des actes administratifs au Chili soit un thème récent, sa présentation et sa synthétisation restent une entreprise relativement délicate. Pour ce faire, il est important de revenir sur les origines de cette règle imposée par la loi de 2003. Le droit administratif chilien ignorait-il tout de la motivation avant cette date? La réponse à cette question est bien évidemment négative, car cette obligation de motivation avait déjà été imposée de manière ponctuelle et parcellaire par des textes et la jurisprudence. Mais la réforme législative de 2003 a permis d'étendre cette obligation à un nombre plus élevé d'actes administratifs et surtout d'en réguler les modalités (I). Sur le plan du principe, l'intervention législative a donc été bénéfique. Pour autant, l'application concrète des dispositions législatives a soulevé et soulève encore quelques interrogations. $\mathrm{Si}$, à certains égards, l'obligation de motivation des actes administratifs semble plus étendue et efficace que celle existant en France, de nombreux doutes subsistent encore dans sa mise en œuvre et il est encore aujourd'hui difficile de voir en elle un principe absolu régissant l'action de l'administration chilienne (II).

\section{Des prémices aux prémisses de l'obligation de motivation des actes administratifs au Chili}

L'obligation de motivation des décisions administratives est relativement récente dans l'histoire administrative chilienne, mais il est possible de remonter aux années 1960 pour en retrouver les premières traces dans des législations ou réglementations spécifiques, puis dans quelques décisions jurisprudentielles $(\mathrm{A})$. Le retour à la démocratie à partir de 1990 aurait pu constituer l'occasion de fonder les nouvelles bases des relations entre État et administrés, et de consacrer ce principe. Cependant, il a fallu attendre 2003 pour que l'obligation de motivation des actes administratifs soit prononcée de manière générale (B).

\section{A. Les origines fragmentaires de l'obligation de motivation}

À la différence d'autres pays hispanophones, le Chili a connu une réception relativement tardive du principe de motivation des actes administratifs ${ }^{18}$. On peut à cet égard considérer que jusqu'en 2003 il y avait un principe d'absence ou d'exceptionnalité de la motivation des actes administratifs ${ }^{19}$ : rares étaient les normes légales ou réglementaires exigeant que l'autorité édicte, dans le cas d'une situation concrète et préalablement définie, un "décret fondé» ou une «décision fondée». L'adjectif «fondé» visait alors à contraindre l'administration à exprimer dans l'acte administratif lui-même les raisons de fait et de droit justifiant la prise de l'acte et donc à le motiver. Ces textes portaient essentiellement sur des matières relatives aux ressources humaines (fonction publique), financières ou matérielles de l'État, ainsi qu'à l'exercice des pouvoirs de sanctions ou disciplinaires ${ }^{20}$. Peut par exemple être cité, parmi d'autres, le décret ayant force de loi du 5 avril 1960 portant le statut administratif des fonctionnaires de l'administration civile, dont l'article 9 prévoyait la possibilité de recruter des non-nationaux à des emplois publics à condition que cette intégration se fasse par un acte administratif motivé ${ }^{21}$. De même, il est possible d'évoquer le décret-loi du 28 novembre 1975 sur l'administration financière de l'État, qui imposait que toute modification du budget en cours d'année soit opérée par décret motivé ${ }^{22}$.

À défaut de texte l'y contraignant, l'administration n'avait aucune obligation d'exprimer dans l'acte luimême les motifs de faits et de droit justifiant sa décision, comme en atteste l'un des premiers avis de la CGR sur cette question:

[...] l'absence de motivation formelle d'un acte administratif ou les erreurs qui peuvent exister à ce sujet, ou lorsque de telles considérations ne sont pas suffisamment complètes n'est pas un défaut qui puisse invalider un acte administratif ou le priver de ses effets. Pour qu'un acte administratif existe, il est exigé qu'existe une cause ou motif qui serve de fondement à l'administration pour agir dans un sens déterminé, mais il n'est pas nécessaire que celle-ci soit formellement exprimée dans l'acte émis,

18. Par exemple, en Argentine, le principe a été consacré par la loi no 19.549 de procédure administrative de 1972.

19. E. Rocha Fajardo, Estudio sobre la motivación..., p. 181.

20. Ibid.; I. Aróstica Maldonado, «La motivación....», p. 502.

21. Décret ayant force de loi $n^{\circ} 368$ du 5 avril 1960 portant statut administratif.

22. Article 14 du décret-loi $n^{\circ} 1.263$ du 28 novembre 1975 relatif à l'administration financière de l'État. Pour d'autres exemples, voir le décret-loi $\mathrm{n}^{\circ} 1.056 \mathrm{du} 7$ juin 1975 ou le décret-loi nº 29.628 du 7 décembre 1976. 
à moins que cela soit expressément exigé, comme lorsqu'il est indiqué qu'un acte déterminé devra être exécuté par décret ou décision fondée ${ }^{23}$.

En l'absence de texte général en la matière, la CGR et les juridictions judiciaires vont toutefois progressivement essayer de donner corps à ces obligations ponctuelles de motivation. Ainsi, dans son avis $\mathrm{n}^{\circ} 56.161$ de 1975 , la CGR considère que l'absence de motivation est un vice pouvant conduire à l'invalidité d'un acte administratif, principalement en matière d'évaluation ou de révocation de fonctionnaires publics, puisque cette exigence était imposée par un décret ayant force de loi. La Cour suprême, quant à elle, vérifiera dès 1969 la qualité de la motivation lorsque celle-ci est exigée puisqu'elle a considéré dans l'arrêt Tobar González que:

S'agissant d'une décision de pouvoir, qui applique une amende administrative, $[\ldots]$ elle ne peut avoir une motivation distincte de celle qui émane des faits déterminés durant l'inspection ${ }^{24}$.

Cette attitude favorable à la motivation se développera à partir des années 1980, comme l'illustre un avis de 1984 de la CGR ${ }^{25}$. Ce dernier a d'ailleurs été analysé $a$ posteriori comme consacrant une exigence généralisée de motivation de tout acte public ${ }^{26}$. Cependant, cette jurisprudence était encore très minoritaire ${ }^{27}$ et surtout hésitante, car les vices de motivation étaient considérés selon les affaires comme des erreurs de fait, des inexistences de motifs, voire des détournements de pouvoir ${ }^{28}$.

Jusqu'en 2003, il était alors justifié de considérer, comme l'a fait Alejandro Vergara Blanco ${ }^{29}$, que, sauf exception, la motivation n'était pas une formalité essentielle de l'acte administratif. Si aucun texte n'imposait une telle obligation, un acte administratif pouvait parfaitement être non motivé, l'administration étant seulement contrainte à présenter la motivation de son acte, en cas de demande ou de recours de la part de l'administré, conformément à l'article 11 bis de la loi sur les bases générales de l'administration de l'État ${ }^{30}$.

\section{B. Les fondements constitutionnels et légaux de l'obligation de motivation}

Comme il a été évoqué précédemment, le retour à la démocratie n'a pas été le déclencheur direct de la consécration de l'obligation de motivation au Chili. Ceci s'explique par le fait que la transition démocratique s'est traduite de manière graduelle en droit. En effet, la fin de la dictature au Chili ne s'est pas accompagnée d'un moment constituant, qui aurait permis d'abroger la Constitution adoptée sous le régime d'Augusto Pinochet en 1980. Pour des raisons politiques, le choix a été fait de maintenir cette Constitution et de l'expurger de certaines de ses enclaves autoritaires. Les deux révisions les plus importantes ont eu lieu en $1989^{31}$ et en $2005^{32}$ et elles ont permis de donner une nouvelle vie à plusieurs articles qui étaient vides de sens sous la dictature, notamment l'article 1 qui dispose à son troisième alinéa que «L'État est au service de la personne humaine et sa finalité est de promouvoir le bien commun ». Cette modification axiologique dans l'interprétation de la Constitution a permis de fournir un cadre favorable à la consécration de l'obligation de motivation des actes administratifs.

Cette consécration viendra du législateur par la loi $n^{\circ} 19.880$ du 29 mai 2003 qui établit les bases des procédures administratives qui régissent les actes des organes de l'administration de l'État ${ }^{33}$. Il s'agit de la première loi de portée générale qui régule la motivation de l'acte administratif au Chili, puisque cette loi s'applique notamment aux ministères, préfectures, services publics créés pour l'accomplissement de la fonction administrative, forces armées, forces d'ordre et de sécurité publiques, gouvernements régionaux ainsi que les municipalités.

Plusieurs articles de cette loi viennent encadrer cette obligation: les articles 11, 16, 35 (alinéa 3) et 41 (alinéas 4 et 6). L'article 11 consacre le principe d'impartialité et, sur cette base, exige que les faits et les fondements de droit soient toujours exprimés dans les actes qui affectent les droits particuliers, soit parce qu'ils limitent, restreignent, privent, perturbent ou menacent leur exercice légitime, ainsi que les actes en réponse aux recours administratifs non contentieux. L'article 16 , quant à lui, consacre le principe de transparence de l'action administrative et affirme que, sauf exception, tous les actes des organes de l'administration de l'État seront publics, y compris leurs fondements. L'alinéa 3 de l'article 35 précise que, dans le cadre d'une procédure administrative, tout rejet d'une preuve apportée par l'administré devra être formulé par un acte motivé. Enfin, l'article 41 dispose que toute décision venant clôturer une procédure administrative devra être motivée (alinéa 4) et que les rapports ou avis qui servent

23. Avis CGR n ${ }^{\circ} 59.671$ du 4 octobre 1961

24. Cour suprême du Chili, 24 avril 1969, Humberto Tobar González, Revista de derecho. Jurisprudencia y ciencias sociales y gaceta de los tribunales, $\mathrm{n}^{\circ} 2,1969$, p. 42.

25. Avis CGR n 33.006 du 21 décembre 1984.

26. A. Fermandois Vöhringer, J. Baraona González, «La inexistencia de los hechos... », p. 87.

27. E. Rocha Fajardo, Estudio sobre la motivación..., p. 189.

28. Ibid., p. 188

29. A. Vergara Blanco, «La motivación...», p. 347-350.

30. Loi organique $n^{\circ} 18.575$ du 5 décembre 1986 portant les bases générales de l'administration de l'État. Voir A. Vergara Blanco, «La motivación...», p. 348.

31. Loi constitutionnelle $n^{\circ} 18.825$ du 17 août 1989 .

32. Loi constitutionnelle $\mathrm{n}^{\circ} 20.050$ du 26 août 2005 .

33. Cette loi fut par la suite révisée par la loi no 20.285 du 20 août 2008 et la loi nº 21.000 du 23 février 2017. 
de motivation devront être incorporés dans la décision elle-même (alinéa 6).

Ce dernier article est à cet égard particulièrement intéressant puisqu'il marque la reconnaissance au Chili de deux types de motivations: les motivations contextuelles et les motivations non contextuelles ou également appelée in aliunde ${ }^{34}$. La motivation contextuelle est celle présente dans le même support matériel que l'acte administratif. La motivation in aliunde, quant à elle, est celle qui est contenue dans un écrit distinct de l'acte administratif, soit dans un acte accessoire à l'acte administratif principal, soit dans un acte édicté dans le cadre d'une autre procédure. Par exemple, la motivation peut être contenue dans un rapport ou un avis, antérieur ou contemporain à l'acte principal. Ceci peut être justifié, au nom de l'unité de la procédure administrative, lorsque les motifs sont complexes et qu'il est plus simple de renvoyer à des actes préparatoires dans lesquels ces motifs ont été spécifiquement analysés. Cependant, afin de garantir le respect de l'article 41 de la loi de 2003, plusieurs conditions doivent être remplies : les documents renvoyés doivent contenir le discours juridico-administratif qui justifie l'édiction de l'acte administratif et non pas uniquement des données brutes; l'acte administratif doit faire une mention explicite et claire de ces documents; et enfin ces documents doivent être placés en annexe ${ }^{35}$. Cette pratique a été reconnue et validée par les tribunaux judiciaires qui vérifient la bonne incorporation de ces motivations à l'acte administratif principal, comme le confirme un arrêt de la cour d'appel de Temuco du 24 novembre $2010^{36}$. Cette interprétation stricte de l'article 41 ferme ainsi toute possibilité à des motivations différées ou à des motivations in aliunde qui viendraient contredire l'acte administratif principal. Comme l'a indiqué la CGR dans un avis de 2008, l'administration ne peut, pour fonder sa décision, ajouter de nouvelles considérations - de fait ou de droit - à travers des documents additionnels ${ }^{37}$.

Les changements introduits par cette loi ont été si importants que certains auteurs de la doctrine chilienne n'hésitent pas à affirmer que la motivation doit aujourd'hui être considérée comme une exigence aussi bien formelle que matérielle des actes administratifs au Chili ${ }^{38}$. Cette interprétation visant à donner la portée la plus large possible à l'obligation de motivation ne repose pas uniquement sur le texte de la loi de 2003 mais sur la Constitution elle-même. Certes, on ne trouve pas dans la Constitution d'obligation expressis verbis de motiver les actes administratifs mais certaines de ses dispositions peuvent permettre de fonder une obligation de motivation. Si certains auteurs la rattachent directement à la démocratie, puisque la motivation serait un moyen de «réalisation du principe démocratique du contrôle diffus et public des agissements des pouvoirs publics par la citoyenneté ${ }^{39}$, d'autres, en revanche, rattachent cette obligation à des articles plus précis. Le principe de transparence et de publicité consacré par l'article 8 de la Constitution est souvent mis en exergue ${ }^{40}$. Il est également possible de le rattacher à l'article $19,2^{\circ}$ de la Constitution qui garantit l'égalité devant la loi et interdit les différences arbitraires ${ }^{41}$. Un autre fondement, qui recueille un plus large consensus, est la protection des droits de la défense des administrés, qui découle lui de l'article 19, $3^{\circ}$ de la Constitution $^{42}$. En effet, la présence d'une motivation permet à l'intéressé de recourir de manière effective contre un acte, soit par le truchement de recours administratifs soit devant une autorité judiciaire. Enfin, plus largement, certains auteurs rattachent cette obligation au passage d'une pratique autoritaire vers un État de droit social, dans lequel l'État est au service de la personne et dont la finalité est le bien commun (article 1 de la Constitution) ${ }^{43}$. En faisant référence à la doctrine américaine de la preferred position relative à la position du citoyen face à l'administration (posición privilegiada), Arturo Fermandois Vöhringer et Jorge Baraona González considèrent que dans le système de droit contemporain «l'autorité doit posséder une raison puissante pour agir sur la sphère de liberté des personnes». Ainsi:

L'exercice de la puissance publique n'est plus considéré comme légitime, mais illégitime à moins que l'on démontre que sa motivation et sa justification sont suffisantes pour intervenir dans le temple de liberté des individus ${ }^{44}$.

34. E. Rocha Fajardo, Estudio sobre la motivación..., p. 108-109.

35. Ibid., p. 111, 112 et 232.

36. Cour d'appel de Temuco, 24 novembre 2010, $\mathrm{n}^{\circ}$ 1.240-2010.

37. Avis CGR n ${ }^{\circ} 7.453$ du 15 février 2008

38. T. Carrera Briceño, «El deber de motivación...», p. 153.

39. E. Rocha Fajardo, Estudio sobre la motivación..., p. 82.

40. Voir, par exemple, E. Rocha Fajardo, Estudio sobre la motivación..., p. 95; A. Parodi Tabak, «Dos sentencias de la Corte suprema: de "punto final" y de "puntos suspensivos" ", in Sentencias destacadas 2010. Una mirada desde la perspectiva de las políticas públicas. Anuario de doctrina y jurisprudencia, Santiago du Chili, LYD, 2011, p. 171; T. Carrera Briceño, «El deber de motivación...», p. 143.

41. E. Rocha Fajardo, Estudio sobre la motivación..., p. 106; T. Carrera Briceño, «El deber de motivación... », p. 145 et 153; A. Fermandois Vöhringer, J. Baraona González, «La inexistencia de los hechos... », p. 82 et 91; A. Parodi Tabak, «Dos sentencias... », p. 170.

42. Voir, par exemple, I. Aróstica Maldonado, «La motivación...», p. 507-508; L. Guzmán Suarez, El control de la discrecionalidad administrativa en Chile, mémoire en droit public, université du Chili, 2001, 187 p., p. 60; A. Vergara Blanco, «La motivación... », p. 345; C. Helfmann Martini, «La motivación como elemento esencial de los actos administrativos y su relación con el principio de razonabilidad», El Mercurio, Opinión, 22 juillet 2016; T. Carrera Briceño, «El deber de motivación...», p. 145.

43. Voir, par exemple, I. Aróstica Maldonado, «La motivación...», p. 506; A. Fermandois Vöhringer, J. Baraona González, «La inexistencia de los hechos...», p. 82; T. Carrera Briceño, «El deber de motivación...», p. 144.

44. A. Fermandois Vöhringer, J. Baraona González, «La inexistencia de los hechos... », p. 8o. 
À la lecture des écrits de la doctrine chilienne, nous serions donc rapidement enclins à accorder aux dispositions de la loi de 2003 une portée extrêmement large. Or, la réalité du droit nous contraint à plus de mesure. D'une part, si la loi de 2003 établit le régime général des actes administratifs au Chili, il importe de souligner qu'elle ne s'applique que de manière supplétoire. Cette loi n'est pas appliquée s'il existe déjà une norme spéciale encadrant l'action administrative ${ }^{45}$ ou si l'application de la loi de 2003 conduit à un détournement de procédure ${ }^{46}$. D'autre part, l'absence d'un nombre élevé de décisions juridictionnelles portant sur ce principe ${ }^{47}$ ne permet pas encore de définir avec clarté l'ensemble des incidences de cette obligation de motivation, laissant plusieurs questions encore en suspens, plus de quinze après son adoption. Et précisément, c'est sur ces doutes qu'il convient de revenir pour mesurer la véritable portée de l'obligation de motivation des actes administratifs au Chili.

\section{Des contours incertains de l'obligation de motivation des actes administratifs}

Un des apports essentiels de la loi de 2003 est qu'elle a permis de généraliser la pratique de la motivation. Si elle constitue aujourd'hui la tendance majoritaire ${ }^{48}$, elle ne s'est pas encore entièrement consolidée. Des interrogations demeurent sur le champ d'application de la loi (A), ainsi que sur les effets à donner au non-respect par l'administration de l'obligation de motivation (B).

\section{A. Un champ d'application aux délimitations encore imprécises}

Loin de consacrer une obligation de motivation pour tous les actes administratifs quels qu'ils soient, la loi de 2003 évoque divers types d'actes soumis à cette obligation. Or, ceux-ci sont évoqués dans différents articles éparpillés dans tout le texte (ce qui ne facilite pas la compréhension), et des contradictions apparaissent.

L'article 11, alinéa 2, de la loi régule deux situations bien spécifiques. Selon cet article, les faits et les fondements de droit devront toujours être exprimés dans les actes qui, soit affectent les droits des particuliers, parce qu'ils limitent, restreignent, privent, perturbent ou menacent leur exercice légitime, soit constituent une réponse finale à une demande de recours administratif non contentieux ${ }^{49}$. Les premiers types d'actes sont appelés les actes d'imposition ou défavorables, lesquels supposent une restriction, privation ou limitation dans le champ juridique d'une personne, en opposition à l'acte administratif favorable, qui produit un élargissement, un agrandissement du patrimoine du citoyen. Les seconds sont ceux qui viennent conclure une procédure administrative récursive déclenchée par une personne intéressée. Ils peuvent parfaitement être favorables ou défavorables à la demande faite par la personne intéressée. Une interprétation littérale et stricte de cet article conduirait à conclure que l'obligation de motivation ne concerne que ces deux types d'actes. A contrario, la motivation ne serait pas exigée pour les actes administratifs favorables, sauf s'ils répondent à un recours administratif ${ }^{5}$.

Néanmoins, cette interprétation entre en contradiction avec les prescriptions de l'article 41, alinéa 4, car celui-ci ne reprend pas cette distinction. Selon l'article 41 , tous les actes administratifs finaux, c'est-à-dire qui viennent clôturer une procédure administrative, quelle qu'elle soit (de recours administratif ou non), doivent être motivés, sans exception, donc y compris les actes administratifs favorables adoptés à l'issue d'une procédure administrative non récursive. Deux options se dessinent alors: soit la loi est incohérente, soit la loi est répétitive et donc les dispositions de l'alinéa 2 de l'article 11 sont superflues. Face à ce doute, la doctrine semble s'accorder pour défendre la deuxième option, afin de donner la portée la plus large possible à l'obligation de motivation. Ainsi, l'article 41 serait celui qui fixe le champ véritable de l'obligation de motivation; l'alinéa 2 de l'article 11 ne servirait qu'à mettre une emphase particulière sur certains types d'actes, voire à inviter le juge à élever son standard de contrôle pour les actes défavorables ou ceux répondant à un recours administratif ${ }^{5}$. Cette interprétation qui vise à donner la plus large portée possible à la loi ne s'appuie malheureusement pas sur une jurisprudence qui permette de la confirmer. Ceci s'explique essentiellement par le fait que la jurisprudence en matière de motivation reste encore assez faible et que les actes administratifs favorables sont encore moins propices à générer du contentieux.

Quoi qu'il en soit, même en adoptant une interprétation large de la loi, des actes administratifs restent exonérés de l'obligation de motivation. De manière certaine, il est possible d'affirmer que sont exclus de son champ d'application les règlements, adoptés à l'initiative de l'administration

45. Voir, par exemple, avis CGR n 11.543 du 23 février 2011; avis CGR no 19.557 du 2 avril 2013. Pour d'autres jurisprudences, voir F. Zúñiga Urbina, C. Osorio Vargas, «Los criterios unificadores de la Corte suprema en el procedimiento administrativo sancionador ", Estudios constitucionales, vol. 14, n' 2, 2016, p. 462 .

46. Voir, par exemple, avis CGR n ${ }^{\circ} 64.580$ du 19 novembre 2009; avis CGR no 72.012 du 19 novembre 2012. Pour d'autres jurisprudences, voir F. Zúñiga Urbina, C. Osorio Vargas, «Los criterios unificadores...», p. 462.

47. C. Helfmann Martini, «La motivación...».

48. E. Rocha Fajardo, Estudio sobre la motivación..., p. 180-181.

49. La loi $\mathrm{n}^{\circ} 19.880$ de 2003 consacre trois recours administratifs ouverts à toute personne intéressée: un recours gracieux (de reposición) qui se fait auprès de l'autorité qui est l'auteure de l'acte, un recours hiérarchique qui se fait auprès du supérieur hiérarchique, et un recours extraordinaire de révision (articles 59 et 60 de la loi).

50. E. Rocha Fajardo, Estudio sobre la motivación..., p. 216.

51. Ibid., p. 217-219 
et dont la formulation générale et impersonnelle ne crée pas de droits aux particuliers. Ensuite, sont également exclus les actes administratifs soumis à une législation particulière, puisque la loi de 2003 est d'application supplétoire ${ }^{52}$. Enfin, nous pourrions être amenés à ajouter à la liste des actes exclus du champ d'application de la loi les actes préparatoires (actos de mero trámite) puisque l'article 41 de la loi de 2003 semble concerner uniquement les actes qui viennent clôturer une procédure administrative, donc ceux qui contiennent la déclaration de volonté d'un organe de l'administration de l'État ${ }^{53}$. Cependant, des exceptions existent. Certaines sont prévues par la loi de manière explicite et d'autres découlent d'une interprétation systémique de la loi. Concernant l'exception prévue explicitement, celle-ci se trouve à l'alinéa 3 de l'article 35 , qui prévoit que, lors de l'instruction d'une procédure administrative, l'administration peut rejeter des preuves apportées par l'intéressé mais uniquement lorsque ces preuves sont manifestement irrecevables ou non nécessaires et elle doit le faire via une décision motivée ${ }^{54}$. Ainsi, bien qu'elle soit un acte préparatoire, la décision de rejet d'une preuve doit être motivée. L'autre exception, quant à elle, résulte d'une interprétation combinée des articles 15 et 41 de la loi. L'article 15 précise qu'a priori seuls les actes finaux peuvent faire l'objet d'un recours (administratif ou contentieux), mais qu'il est possible d'intenter un recours (administratif ou contentieux) si des actes préparatoires déterminent l'impossibilité de continuer une procédure ou s'ils produisent un déni de justice. Dès lors, en reconnaissant que certains actes préparatoires peuvent mettre fin à une procédure et qu'ils ont un effet décisoire suffisant pour justifier un recours contre eux, une interprétation systémique de la loi devrait conduire à considérer que ces actes préparatoires sont également soumis à l'obligation de motivation ${ }^{55}$.

Ces quelques exemples montrent à quel point le champ d'application de la loi de 2003 reste encore indéterminé. Il y a clairement une possibilité de donner à cette loi une portée extrêmement large, mais, en l'absence de jurisprudence constante confirmant cette interprétation, elle reste encore hypothétique.
L'autre incertitude laissée par la loi est celle relative à la forme exacte de cette motivation. Quel type de motivation impose la loi? Quel serait l'acte qui ne respecte pas son obligation de motivation? La loi n'apporte pas de réelle précision sur ce point. De manière simple, et en adoptant une définition très réduite de la motivation, telle que celle développée par Iván Aróstica Maldonado, selon laquelle la motivation est uniquement l'expression des motifs dans l'acte administratif lui-même ${ }^{56}$, on pourrait considérer que l'acte qui ne respecte pas l'obligation de motivation est celui qui n'a pas extériorisé les motifs de fait et de droit qui ont justifié son édiction, c'est-à-dire lorsqu'il y a un défaut total de motivation ${ }^{57}$. Ce vice constituerait le vice de motivation par excellence. Cependant, la majorité de la doctrine considère que la motivation est plus qu'une simple extériorisation des motifs. Comme le souligne Eduardo Soto Kloss, la motivation est «la justification normative, factuelle et rationnelle de la décision que l'autorité administrative adopte pour satisfaire une nécessité publique concrète $»^{58}$. La motivation doit donner acte du "processus de qualification juridique [des] faits dans la norme juridique» ainsi qu'expliquer «le raisonnement suivi pour arriver à la décision comme conséquence d'un processus logique et juridique $»^{59}$. Les motivations doivent donc être suffisantes: l'acte administratif doit se suffire à lui-même ${ }^{60}$ et contenir tous les éléments nécessaires à sa compréhension de manière claire, ce qui exclut toute motivation tronquée ou partielle. Concernant les éléments de droit, le simple signalement (visa) des normes légales applicables n'est pas considéré comme suffisant aux yeux de la doctrine chilienne. Les normes légales doivent être explicitement citées, accompagnées d'une analyse de la situation, et présentées de façon à justifier le contenu ou l'objet de la décision ${ }^{61}$. Mais, au-delà, les motivations doivent également éviter l'utilisation d'expressions vagues, génériques ou vides de sens car passe-partout ${ }^{62}$. Cela n'implique pas que la motivation soit nécessairement longue. Elle peut être succincte, à partir du moment où elle est suffisamment indicative, c'est-à-dire qu'elle expose les éléments nécessaires pour que le destinataire comprenne l'acte ${ }^{63}$.

52. Certaines de ces lois prévoient également une obligation de motivation pour ces actes spécifiques. Par exemple, les contrats administratifs sont régis par la loi-cadre $\mathrm{n}^{\circ} 19.886$ sur les contrats administratifs de fourniture et de prestation de services du 30 juillet 2003 . L'article 10 , alinéa 1 établit que: «L'adjudication du contrat s'opère via une décision motivée de l'autorité compétente». Sur le contrôle opéré par la CGR sur les règles relatives au contrat administratif, voir J. L. Lara Arroyo, C. Helfmann Martini, «La contribución de la jurisprudencia contralora al desarrollo y delimitación de los principios de la contratación administrativa ", in La Contraloría General de la República. Conmemoración por su 85² Aniversario de vida institucional (1927-2012), p. 439-461.

53. E. Rocha Fajardo, Estudio sobre la motivación..., p. 224-225.

54. Confirmé par l'avis CGR no 39.348 du 30 août 2007.

55. E. Rocha Fajardo, Estudio sobre la motivación..., p. 225-226.

56. I. Aróstica Maldonado, «La motivación...», p. 500.

57. Ibid. ; A. Fermandois Vöhringer, J. Baraona González, «La inexistencia de los hechos... », p. 91.

58. E. Soto Kloss, «La fundamentación del acto administrativo en la jurisprudencia de los tribunales ordinarios de justicia», Derecho público iberoamericano, $\mathrm{n}^{\circ} 13,2018$, p. 226.

59. T. Carrera Briceño, «El deber de motivación...», p. 153.

60. E. Rocha Fajardo, Estudio sobre la motivación..., p. 119; A. Parodi Tabak, «Dos sentencias... », p. 173; J. F. García, « Corte suprema y motivación del acto administrativo (a propósito de la sentencia Rectora Universidad de Aysén)», El Mercurio, Constitucional, 10 octobre 2017.

61. E. Rocha Fajardo, Estudio sobre la motivación..., p. 120-122.

62. Ibid., p. 119; E. Soto Kloss, «La fundamentación... », p. 228.

63. L. Guzmán Suarez, El control de la discrecionalidad..., p. 6o-61; A. Parodi Tabak, «Dos sentencias...», p. 173; E. Rocha Fajardo, Estudio sobre la motivación..., p. 124. 
Cette exigence qualitative de la motivation est confirmée par la jurisprudence. S’il existe des jurisprudences isolées dans lesquelles une motivation des plus sommaires a été considérée comme suffisante ${ }^{64}$, la majorité des arrêts atteste d'une exigence élevée en matière de qualité de motivation ${ }^{65}$. Dans un arrêt du 2 décembre 2014, la Cour suprême a par exemple considéré que:

L'acte administratif doit contenir les fondements sur lesquels il se fonde afin de légitimer la décision prise par l'autorité, ces raisons ne peuvent être simplement formelles car elles tomberaient dans la catégorie des raisons arbitraires et, donc, illégales ${ }^{66}$.

Dans un arrêt du 19 juin 2017, Rectora Universidad de Aysén ${ }^{67}$, la Cour a précisé son analyse en indiquant qu'une motivation devait toujours inclure:

[...] une relation circonstanciée des fondements de la décision de façon à ce que puisse être attestée la rationalité intrinsèque, c'est-à-dire la cohérence avec les faits déterminants et la finalité publique qui doit être poursuivie.

Cette affaire est d'autant plus intéressante que l'acte administratif en question était un décret qui avait déjà fait l'objet d'un contrôle ex ante par la CGR, laquelle avait considéré qu'il satisfaisait les standards en matière de motivation ${ }^{68}$. Quelques mois plus tard, la Cour suprême pousse encore davantage son exigence, en considérant que «la motivation d'un acte administratif [...] doit [...] être adaptée à la finalité poursuivie dans son édiction ${ }^{69}$, lui permettant ainsi d'opérer un contrôle de «nécessité» et $\mathrm{d}^{\prime}$ "idonéité» de cette motivation ${ }^{70}$. Cette exigence de motivation idoine se retrouve même dans des contentieux où habituellement l'État dispose d'une grande marge d'appréciation: celui des visas. Dans une affaire tranchée en 2018, un citoyen pakistanais s'était vu refuser son visa par l'autorité consulaire «en raison de convenance ou utilité nationales ». La Cour suprême a considéré que ce seul argument ne constituait pas une motivation suffisante, s'il n'était pas accompagné par des circonstances factuelles ${ }^{71}$.

De son côté, la CGR, même si elle peut avoir des appréciations divergentes sur certains textes, est particulièrement exigeante en ce qui concerne certains types d'actes administratifs, notamment les actes dits de qualification, c'est-à-dire les actes d'évaluation des fonctionnaires publics. Ces actes ne sont pas encadrés par la loi de 2003 mais par une norme légale spécifique ${ }^{72}$. La CGR exige que tous les actes entourant cette évaluation (appelée qualificación) soient motivés de façon à ce qu'il y ait une concordance entre le fondement émis et les notes assignées à l'employé et que l'employé soit en condition d'assumer de manière adéquate sa défense afin de contester sa qualification ou qu'il soit en mesure d'améliorer son comportement de travail pour la période suivante, spécialement dans des aspects pour lesquels a été signalée une baisse de son évaluation ${ }^{73}$.

Au regard de cette jurisprudence de la Cour suprême et de la CGR, il semblerait que toute motivation doit contenir les éléments suivants ${ }^{74}$ : les faits qui sont donnés comme établis, le sens de la norme appliquée, la qualification juridique des faits, ainsi que les raisons qui ont mené l'administration à considérer ou non les allégations des intéressés (elle doit reprendre les arguments de l'administré et y répondre en argumentant). Toutefois, dans les faits, il est difficile d'établir des paramètres ou critères précis d'une motivation suffisante. La théorisation d'un «administré-moyen» qui serait en capacité de comprendre la motivation est une entreprise difficile et risquée ${ }^{75}$. D'autant plus que la motivation est une justification qui dépend d'une réalité et d'un contexte donné, qu'il s'agisse du lieu, du moment, de l'expertise de l'organe administratif, du type de décision administrative dont il est question (acte discrétionnaire ou de compétence liée, acte défavorable ou favorable, acte qui répond positivement à un recours ou qui le rejette, etc.) : ainsi il apparaît difficile d'établir un cadre juridique unitaire pour décrire les irrégularités d'une motivation ${ }^{76}$.

\section{B. Des incidences de l'obligation de motivation encore flottantes}

Si, seize ans après l'entrée en vigueur de la loi de 2003, il existe encore des doutes sur le champ d'application exact

64. On peut par exemple citer ici un jugement du Tribunal du travail (juzgado del Trabajo de Santiago), qui le 23 mars 2009 a considéré qu'on ne pouvait pas dire que la décision de la Direction du travail n'était pas motivée, car «ceci serait le cas s'il s'agissait d'une décision pure et simple, qui n'invoque aucun antécédent, ce qui n'est pas le cas", alors que, dans cette affaire, l'administration en cause s'était contentée d'incorporer dans la décision, en guise de motivation, un formulaire pré-imprimé rempli avec les informations de base de l'affaire, comme le nom des parties, une liste d'antécédents, les demandes formulées par l'intéressé et la législation applicable, sans plus de développement.

65. La jurisprudence des tribunaux judiciaires est considérée sur cet aspect relativement homogène; voir E. Soto Kloss, «La fundamentación... », p. 226.

66. Cour suprême du Chili, 2 décembre 2014, Herrera Jiménez c. Comisión de Libertad Condicional, no 27.467-2014.

67. Cour suprême du Chili, 19 juin 2017, Rectora Universidad de Aysén, nº 3.598-2017.

68. Avis CGR no 59.669 du 11 août 2016.

69. Cour suprême du Chili, 21 septembre 2017, Aes Gener SA c. Municipalidad de Tocopilla, nº 7.025-2017.

70. E. Soto Kloss, «La fundamentación... », p. 229.

71. Cour suprême du Chili, 26 avril 2018, Kashib Nawab, nº 7.501-2018.

72. La loi $n^{\circ} 18.839$ du 23 septembre 1989 portant statut administratif, refondue par le décret ayant force de loi $n^{\circ} 29$ du 16 juin 2004 .

73. Avis CGR no 16.292 du 5 avril 2005. Voir également avis CGR no 36.148 du 7 mai 2015; avis CGR no 45.494 du 8 juin 2015 . Pour plus de jurisprudence et d'explication autour de ce processus de qualification, voir T. Carrera Briceño, «El deber de motivación...», p. 143-155.

74. E. Rocha Fajardo, Estudio sobre la motivación..., p. 124; A. Parodi Tabak, «Dos sentencias... », p. 176.

75. E. Rocha Fajardo, Estudio sobre la motivación..., p. 123.

76. L. Guzmán Suarez, El control de la discrecionalidad..., p. 62. 
de l'obligation de motivation, des interrogations encore plus profondes demeurent concernant les effets à donner en cas de non-respect de cette obligation de motivation.

Une des principales raisons de cette imprécision provient du fait que l'ordonnancement juridique chilien n'énonce pas de manière systématique les vices des actes administratifs ni les traite de manière détaillée. L'autre raison réside quant à elle dans ce contentieux administratif particulièrement complexe et alambiqué, présenté en introduction. Ce contentieux étant construit sur la base de différents recours, chacun ayant ses propres règles procédurales, ses propres critères et ses propres effets, il apparaît bien difficile de pouvoir élaborer une présentation claire et synthétique des conséquences d'un acte administratif non ou mal motivé

À première vue pourtant, la loi de 2003 semble nous apporter des éléments de clarté sur cette question, dans la mesure où elle indique à son article 13 quels sont les effets à donner à un vice de forme entachant un acte administratif. Selon cet article, le vice de procédure ou de forme affecte la validité de l'acte administratif uniquement lorsqu'il atteint une des exigences essentielles de la validité de l'acte, soit par sa nature, soit par une exigence de l'ordre juridique, et qu'il génère un préjudice à l'intéressé; les deux conditions étant cumulatives. Ainsi, conformément à cet article, tous les vices de forme n'entraînent pas systématiquement l'illégalité de l'acte, seuls ceux ayant les conditions susmentionnées pourront avoir un tel effet. Dans la mesure où la motivation est bien souvent rangée dans la catégorie des vices de forme, afin de la distinguer des motifs, le vice de motivation ne devrait conduire à l'annulation de l'acte administratif que lorsque lui aussi génère les conséquences déjà évoquées.

Cette clarté s'obscurcit pourtant lorsque l'on examine la doctrine sur cette question. Certains réfutent cette interprétation parce qu'ils considèrent que le défaut ou le manque de motivation ne doit pas être rangé dans la catégorie des vices de forme mais dans les vices plus substantiels. Puisque la motivation constitue une garantie de plusieurs principes constitutionnels (principe de transparence, droits de la défense ou interdiction de l'arbitraire), son absence ou son insuffisance devrait conduire nécessairement à l'annulation de l'acte ${ }^{77}$, surtout lorsque l'acte est un acte discrétionnaire. À l'appui de cette thèse on peut mettre en avant certains avis de la CGR, qui font explicitement référence à la Constitution. Par exemple dans son avis du 29 janvier 2008, la CGR a considéré que:
[...] la motivation est un élément essentiel pour garantir la juridicité de l'acte [administratif], conformément aux articles 6 et 7 de la Constitution politique, et le principe d'interdiction de l'arbitraire, selon les prescriptions de l'article $19,2^{\circ}$, de la même Constitution ${ }^{78}$.

De même, plusieurs arrêts de la Cour suprême insistent sur la nature arbitraire d'un acte administratif mal ou non motivé ${ }^{79}$. Ainsi, le vice de motivation ne pourrait constituer qu'un simple vice de forme mais serait également un vice d'arbitraire, un vice affectant la légitimité du contenu de la décision administrative ${ }^{80}$. D'autres auteurs refusent également d'accorder à la motivation une portée purement formelle, en s'appuyant cette fois-ci sur la loi de 2003 elle-même. Dans la mesure où l'article 41 de la loi consacre la motivation comme un élément exigé de tout acte administratif venant clôturer une procédure administrative, il est possible de considérer que toute motivation est nécessairement un critère essentiel de l'acte administratif et dont l'absence ou l'insuffisance devrait entraîner mécaniquement l'annulation de l'acte ${ }^{81}$. Toutefois, il est possible d'avoir une position plus nuancée et de concilier ces points de vue en estimant que la motivation est une exigence certes formelle, mais que certaines motivations présentent un caractère essentiel et que ce n'est que lorsque cette motivation porte un préjudice à l'intéressé que l'acte administratif sera annulé.

Le dernier aspect intéressant concernant les vices de motivation dans le droit administratif chilien est la question des incidences pratiques qu'il convient de tirer d'une annulation. Le contentieux en offre plusieurs exemples. Il est possible d'évoquer l'arrêt de la Cour suprême du 8 janvier 2019, dans lequel la Cour avait statué sur l'illégalité d'un acte de la Direction des œuvres hydrauliques qui mettait fin de manière anticipée à l'emploi d'un fonctionnaire dans son service du fait d'une motivation insatisfaisante ${ }^{82}$. L'illégalité de l'acte a conduit la Cour suprême à prononcer l'annulation de l'acte, à exiger que le requérant soit réincorporé dans ses fonctions jusqu'au 31 décembre 2018 (fin prévue de sa mission dans le service) et qu'il reçoive toutes les rémunérations non perçues ${ }^{83}$. Cependant, dans d'autres affaires, un peu antérieures, la Cour a semblé plus hésitante, préférant parfois laisser l'administration tirer les conséquences pratiques de l'illégalité de l'acte. Pour l'illustrer il est possible de faire une comparaison entre deux arrêts qui portaient tous deux sur des amendes prononcées par l'administration ${ }^{84}$. Les personnes concernées avaient fait un recours auprès de l'administration mais celle-ci n'avait

77. A. Parodi Tabak, «Dos sentencias... », p. 176-177; A. Fermandois Vöhringer, J. Baraona González, «La inexistencia de los hechos...», p. 90 et 91.

78. Avis CGR no 4.168 du 29 janvier 2008.

79. Voir, par exemple, Cour suprême du Chili, 26 mars 2018, Cruz Zamora c. Municipalidad de Pedro Aguirre Cerda, nº 43.400-2018; Cour suprême du Chili, 8 janvier 2019, Leonel Fernando Claros Díaz c. Dirección de Obras Hidráulicas, n 20.768-2018.

80. E. Rocha Fajardo, Estudio sobre la motivación..., p. 272.

81. Ibid., p. 262.

82. Cour suprême du Chili, 8 janvier 2019, Leonel Fernando Claros Díaz...

83. Pour un exemple similaire au niveau des effets, voir Cour suprême du Chili, 26 mars 2018, Cruz Zamora...

84. Cour suprême du Chili, 6 janvier 2010, Panificadora Lo Saldes Limitada c. Dirección regional del trabajo de la región metropolitana, nº 8.445-2009; Cour suprême du Chili, 15 juin 2010, Redbus Urbano SA c. Inspección comunal del trabajo de Santiago oriente, n ${ }^{1.764-2010 .}$ 
pas accédé à leur demande. La Cour suprême considéra dans les deux cas que les refus des demandes avaient été mal motivés et avait donc prononcé leur annulation. Mais alors que dans l'arrêt du 6 janvier 2010, la Cour décide elle-même de se prononcer sur le fond de la demande et ordonne de laisser sans effets les amendes prononcées par l'administration, dans l'arrêt du 15 juin 2010, elle se limite à inviter l'autorité administrative à se prononcer sur le fond concernant les amendes, au risque donc que cette autorité prenne la même décision avec une nouvelle motivation $^{85}$.

Divers points d'incertitudes entourent donc encore le devoir de motivation des actes administratifs au Chili. Or, il y a fort à parier que ces incertitudes demeureront encore pendant un certain temps. D'une part, le maintien d'une structure assez complexe en matière de contentieux administratif et l'obsession bien ancrée de la doctrine chilienne du droit étranger contribueront à alimenter les désaccords ou les décalages et constitueront un frein durable à une lecture unifiée du droit administratif chilien, recentré sur ses spécificités. D’autre part, les caractéristiques propres à la motivation impliquent la rémanence d'une certaine indétermination, du fait des effets synergiques existant entre les vices des actes administratifs. Lorsqu'un acte est vicié par une irrégularité, dans la grande majorité des cas, l'acte ne comporte pas ce seul vice, mais il en existe d'autres qui lui sont potentiellement connectés et reliés ${ }^{86}$. Dans le cas de la motivation, en tant qu'expression de la logique administrative, rares sont les cas où les irrégularités de la motivation sont les seules irrégularités. Il apparaît donc difficile de l'isoler et de l'analyser en excluant les contingences mêmes de l'acte et de ses autres vices.

Cependant, au regard de ce tableau quelque peu sombre, il importe d'y apporter quelque peu de clarté. La consécration générale, et pas seulement ponctuelle, de l'obligation de motivation au Chili a grandement participé à améliorer la qualité des décisions administratives. Surtout, elle a permis d'introduire l'idée d'une relation plus égalitaire et plus démocratique avec les administrés, et donc d'écrire une nouvelle page de l'administration post-dictatoriale. 